\title{
How we prepared our operating theatre for patients with SARS-CoV-2 virus
}

\author{
Justyna Rymarowicz, Michał Pędziwiatr, Piotr Major, Michał Nowakowski \\ $2^{\text {nd }}$ Department of General Surgery, Jagiellonian University Medical College, Krakow, Poland
}

Videosurgery Miniinv 2021; 16 (1): 117-122

DOI: https://doi.org/10.5114/wiitm.2020.95090

\begin{abstract}
The COVID-19 disease continues to cause a global pandemic. The University Hospital in Krakow has been designated as one of the COVID-19 hospitals. To prepare for the pandemic we needed to implement strategies that would protect the health care workers, reduce in-hospital transmission, and provide optimal care for the patients. In the operating department, these preparations involve the cooperation of multiple teams and can pose significant difficulties. Here, we describe measures taken in response to the COVID-19 outbreak. These include, adjustments made in OR setups, modification of workflow and processes, and the introduction of adequate personal protective equipment. We believe that these containment measures are required in order to provide an adequate quality of care to COVID-19 patient and to minimise the risk of cross-infection to staff members and other patients.
\end{abstract}

Key words: emergency surgery, personal protective equipment, COVID-19, coronavirus disease, operating department, theatre staff.

COVID-19 is an infectious disease caused by SARS-CoV-2 [1]. Its clinical manifestations are very similar to those of Severe Acute Respiratory Syndrome SARS-CoV-1 and most commonly include fever, dry cough, and dyspnoea [2]. Although the majority of infected individuals display mild or no symptoms, a portion progress to acute respiratory distress syndrome with a high fatality rate [2].

The first cases of the novel coronavirus were reported in Wuhan, Hubei province, China in December 2019 [3]. Since then the virus SARS-CoV-2 rapidly spread to other countries. By the 10 April 2020 it had been reported in 185 different countries [4]. Its spread to Poland occurred on the $24^{\text {th }}$ of February. At the time of this review (10.04.2020) there are 5742 confirmed cases in Poland and the number is expected to grow [4].

To control the epidemic, the Ministry of Health initiated its National Strategy. One aspect is the introduction of a chain of nationwide Communicable Disease Centre Hospitals. Our hospital has been designated as one of them. Given the exponentially growing number of people infected, some COVID-19-positive patients are likely to require surgical intervention during this pandemic.

The operating theatre environment is at particular risk of transmitting COVID-19 because high-risk procedures such as airway manoeuvres and aerosolgenerating procedures are performed there $[5,6]$. It mandates extraordinary precautions in managing perioperative care of patients with suspected or confirmed infection with COVID-19.

This article presents strategies implemented in our operating department (OD) to help prevent cross-infection during surgery.

Our hospital is a major surgical, cancer, and trauma centre consisting of 20 operating rooms (OR) de-

\section{Address for correspondence}

Assoc. Prof. Michał Nowakowski MD, PhD, $2^{\text {nd }}$ Department of General Surgery, Jagiellonian University Medical College, 2 Jakubowskiego St, 30-688 Krakow, Poland, e-mail: m.nowakowski@uj.edu.pl 
signed in a circular order. Figure 1 shows the plan of the OD. A thorough analysis of the recent guidelines published by EAES, SAGES, and the Spanish Association of Surgeons was undertaken in order to create a designated COVID Operating Area (COA) [6-9]. A team composed of representatives of all major stakeholders including surgeons, anaesthesiologists, nurse anaesthetists, theatre nurses, technicians, epidemiologists, management, cleaning, infrastructure, and maintenance personnel was created. Additionally, we consulted our close co-operators in intensive care unit (ICU), surgical wards, and supply chains. All work was widely conferred with operating theatre staff, and simulation tests were performed for all major steps.

The three sequential ORs located close to the fast-track elevator were selected for the COA. The first operating room was dedicated to general surgery and polytrauma patients, the second was dedicated to orthopaedic trauma cases, and third to neurosurgical cases. Equipment necessary for all other surgical specialties was prepared for delivery to the first OR. This created a versatile, multipurpose unit capable of accommodating every emergency procedure we typically perform in our hospital, with up to three emergency procedures carried out simultaneously.

The COA, which forms a separate fire area in the operating department, was divided into five distinct zones. Each zone was intuitively colour coded, and marking signs were placed on the entrance and exit.
The red zone contains operating rooms, the orange zone contains scrub rooms, the yellow zone contains the corridor connecting the red and the green zone, the violet zone contains an area where access is strictly forbidden, and the green zone contains the remaining, uncontaminated part of the operating department. The scheme below illustrates how the zones were divided in the COA with the patient transfer route and the staff flow (Figure 2).

All COVID-positive patients from the ICU, the emergency department (ED), or COVID-19 wards can access the COA via the fast-track elevator, which was specially reassigned for SARS-CoV-2 positive patients. The personnel involved in transfer wear full personal protective equipment (PPE) and the patient either wears a face mask or is intubated if coming directly from the ICU or ED. In this way, we created a separate passage for all COVID-positive patients requiring transfer to the $O D$.

The positive pressure environment in the operating suites continues in the COA because our operating rooms were designed in this manner. Laminar flow with positive pressure in the OR is in our opinion much safer than negative pressure, which is difficult to predict. There is a very common misconception that what makes a negative pressure room is a change in the pressure alone. However, negative and positive pressure rooms are designed in two different ways. The negative pressure room needs to be airtight to prevent an uncontrolled air entry, and it does not secure the sterility of the surgical field. The

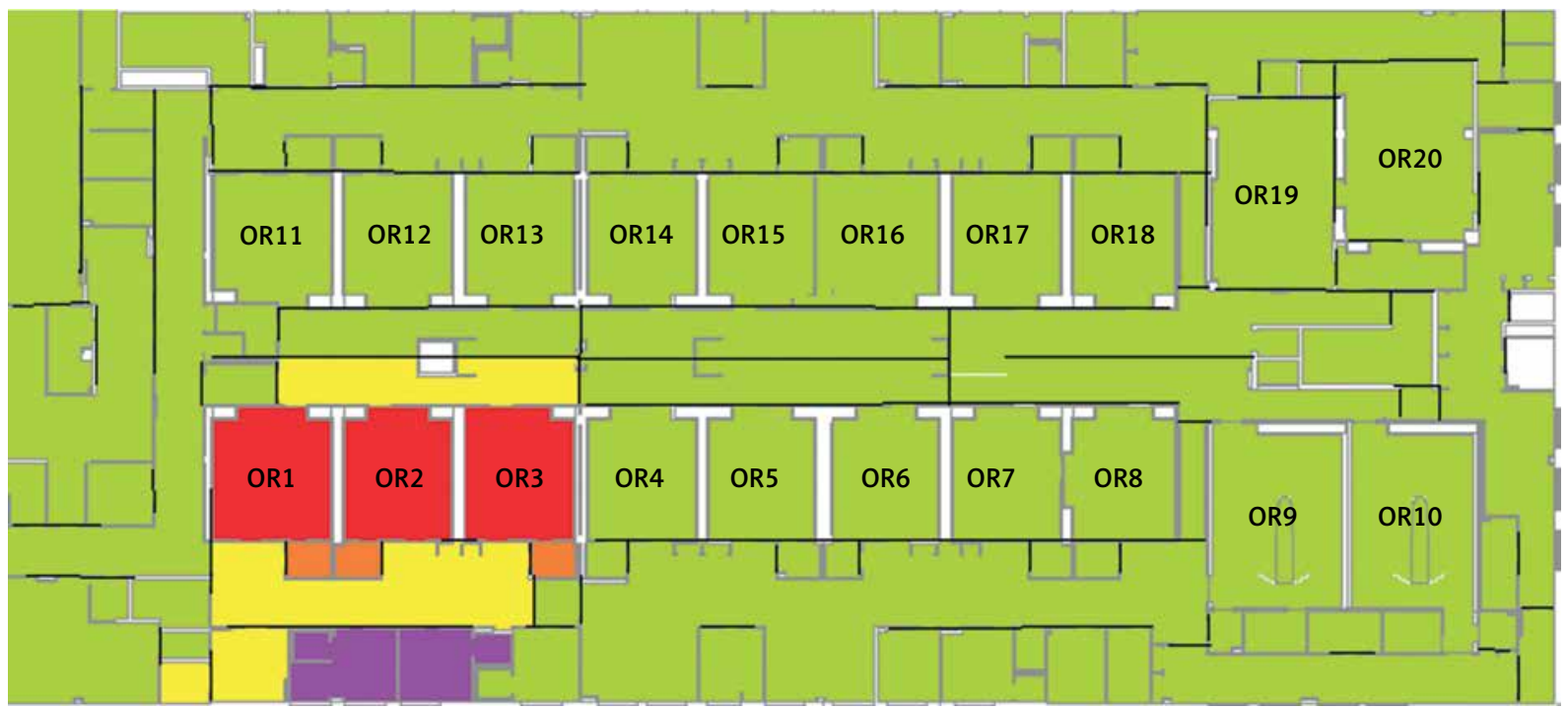

Figure 1. Operating department layout plan 


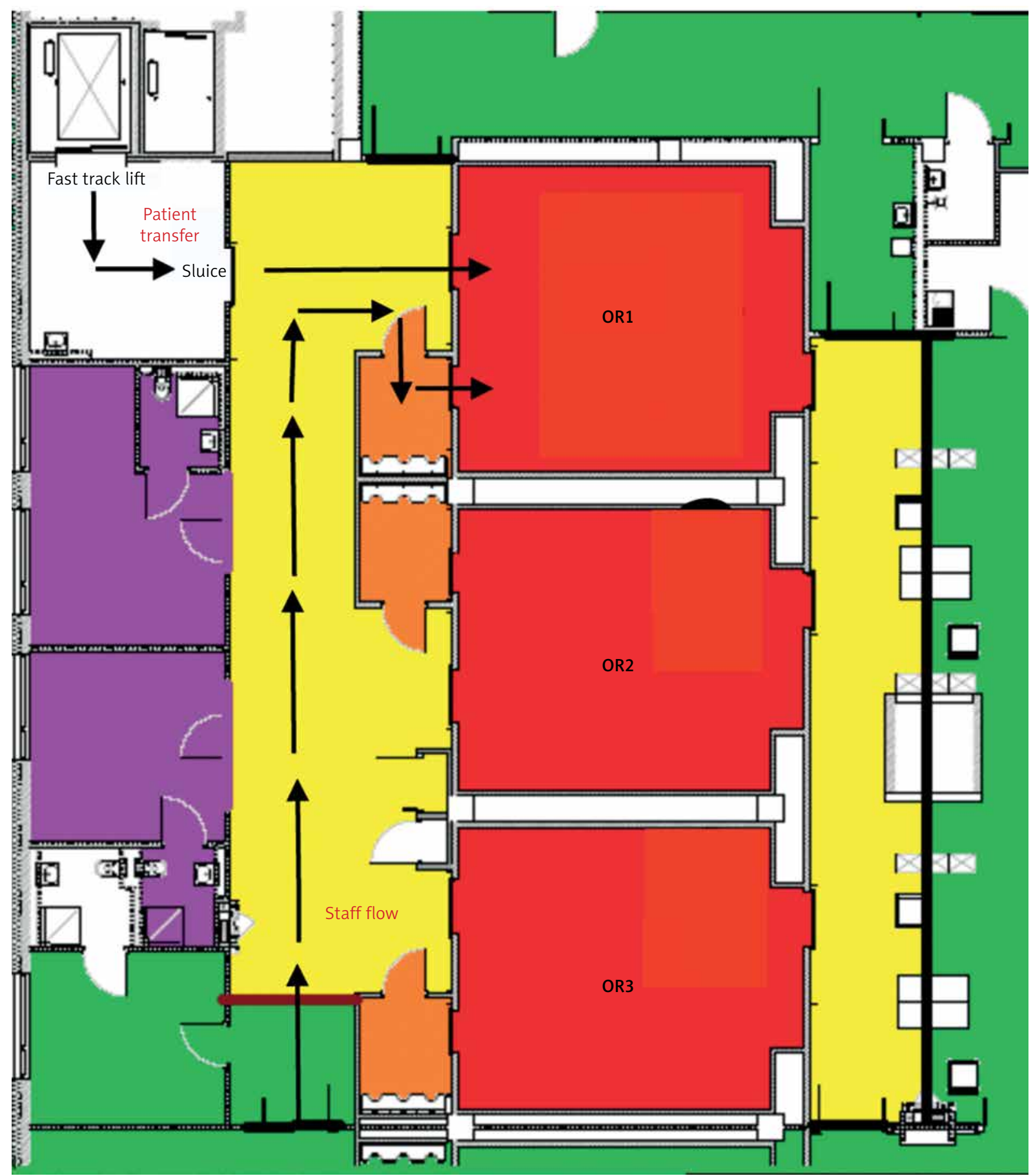

Figure 2. COVID Operating Area layout plan with patient and staff flow

positive pressure operating rooms are not airtight, and by employing technologies such as laminar air flow they limit the exposure of the sterile field to external contamination. Furthermore, in a standard OR there are multiple technical entry channels for gas pipes and electrical cables, and a great deal of empty space above the ceiling and in the walls. Allowing polluted and contaminated air to enter the operating facilities by shifting the pressure gradient would contaminate the sterile surgical field and pose an unpredictable risk to the patient and the staff members. To our knowledge, there are not suf- 
ficient data to discard a high-volume laminar flow in the COA. However, the pressure gradient between the OR and the surrounding area was reduced and the air exchange cycle rate was increased [10]. Additionally, the yellow zone is separated from the green area by fire-resistant doors and concrete walls. The walls have very limited air permeability, hence we assumed that any air contamination is limited to the yellow zone.

In the COA, all nonessential portable devices are removed. The stationary equipment and that necessary for the procedure are wrapped with cling film. An airway trolley, with minimal but adequate stock, is provided. Surgical instrument sets for the most frequently performed emergency operations were pre-emptively prepared and stored outside the COA. Whenever possible, disposable tools are used.

There are designated team members allocated for each zone daily. All theatre staff are screened for COVID-19 symptoms with a personal questionnaire and each member measures their temperature twice daily, and in case of fever they contact the infectious control team for further guidance. Additional temperature checks are performed by a designated staff member on entry to the OD.

Before the start of each operation the theatre coordinator is informed and adequate surgical and anaesthesia equipment is delivered to the operating room. The COA team members put on full PPE in the green zone. No personal items, including cell phones, keys, entrance cards, and piercing jewellery are al- lowed beyond the green area. The green zone coordinator is accountable for supervising the rest of the team during donning, and every person is checked before entering the yellow zone. Figure 3 depicts PPE elements for each team member.

The anaesthetic team, consisting typically of one senior anaesthetist and one anaesthetic nurse, enter the yellow zone first. However, an additional anaesthetic team member is allowed for unstable or polytrauma patients. They are responsible for making sure that all the necessary equipment is prepared, the entrance to each zone is closed, and no unassigned staff are present. Only then can they receive the patient from the sluice and transfer to the OR. If required, endotracheal intubation is performed in the OR. The airway should be secured with the method with the greatest chance of first-time success, to avoid repeated instrumentation of the airway. If needed, endotracheal tube with a pre-mounted bougie and heat and moisture exchanger filter, fibreoptic bronchoscope, and single-use video-laryngoscope are available. Only after the airway is secured are the surgical team allowed to enter the OR following the same donning protocol. The team normally consists of two surgeons and two theatre nurses. Care is taken to ensure that the most experienced personnel are selected to provide optimal care and minimise operating time. In terms of the right surgical approach, the surgeon's experience and the patient's condition play a major role in a decision-making process. Our aim is to provide

PPE for anaesthetic team member

PPE for the non-sterile theatre team member

PPE for the sterile surgical team member

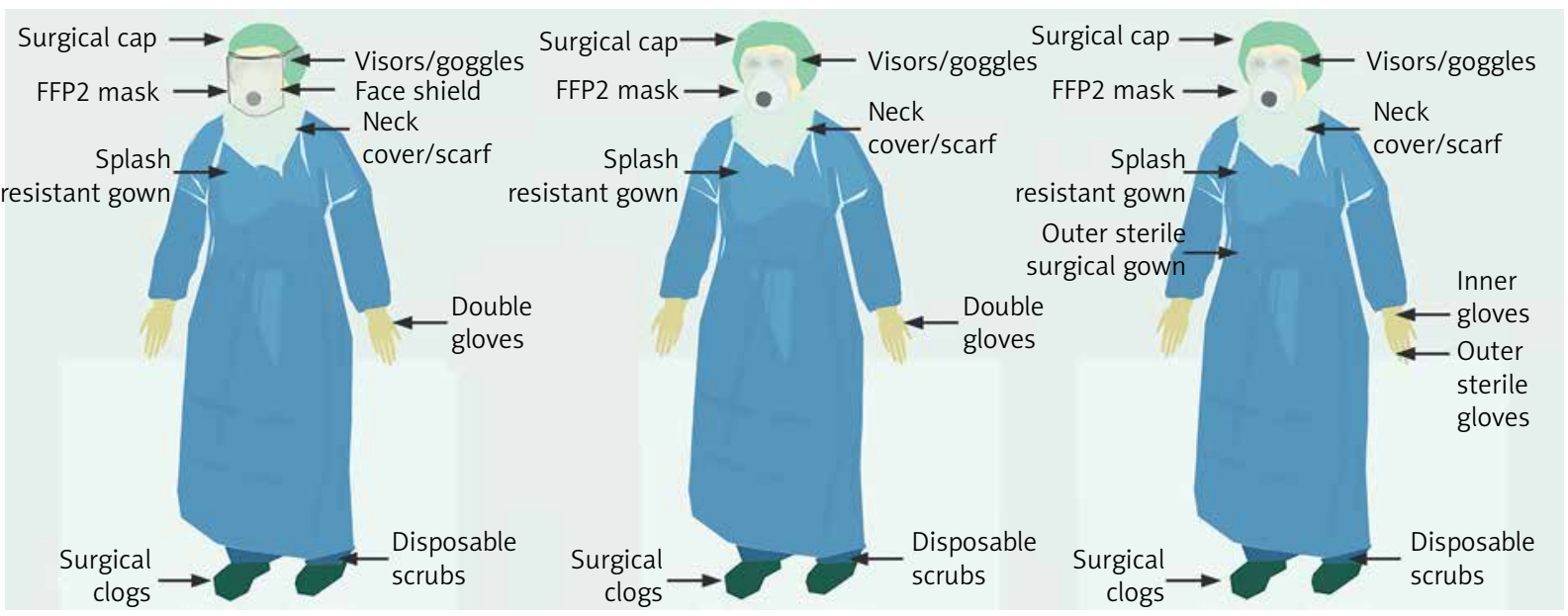

Figure 3. Personal protective equipment elements for each team member

$P P E$ - personal protective equipment. 
the best quality of care to patients and to maintain their safety. The laparoscopic approach shortens the length of in-hospital stay and decreases the risk of infectious complications [11, 12]. Because there are not sufficient data to discard laparoscopic surgery, it is our preferred approach whenever feasible. However, care should be taken to minimise the possibility of inadvertent release of pneumoperitoneum, by using adequately air-tight trocars and maintaining the intraabdominal pressure between 8 and $11 \mathrm{~mm} \mathrm{Hg}$ [7-9]. Likewise, smoke evacuators are utilised whenever possible, and surgical personnel are made aware that aerosol-generating procedures may pose a higher risk. In a typical setting, a total of six staff members are present in the red zone. It is crucial to keep the number of people present in the OR to a minimum to facilitate efficient communication, reduce the number of health care workers potentially exposed, and curtail the use of PPE [6].

During the surgery, the theatre coordinator located in the green zone has direct visual control of the OR through an OR video camera and direct voice contact via a wall-mounted loudspeaker OR phone. The coordinator's main role is to organise additional equipment or personnel that might be required in the red zone. They are supported by a designated theatre nurse, an anaesthetist nurse, and an anaesthesiologist. In the yellow area stations a runner, donned in full PPE, transfers the supplementary equipment from the green zone and places it on a tray located in the orange zone. Special care is taken to minimise the transfer of items from the red zone outside, but there are no limits to the transfer of equipment or materials to the red zone. The doors of the OR are kept closed at all times. No one is allowed to leave the OR until surgery is finished, unless there is a threat to their health or life.

At the end of the procedure, the surgical team exits the OR before the patient is extubated. They discard their sterile gowns and external gloves, change theatre clogs, and perform hand hygiene techniques in the orange zone before moving into the yellow area. At the exit of the yellow area the splash-resistant gown and visors are removed, the theatre clogs are changed again, and hand hygiene is performed. In between the yellow and green zone there is a sluice with showers where personnel remove the FFP2 masks, discard disposable scrubs, shower, and wear new scrubs including new theatre clogs. After that, they can enter the green area and are able to leave the department. Clear instructions are placed on walls and doors on each exit point to facilitate PPE doffing. In addition, the yellow zone runner is responsible for helping other team members to doff correctly.

For patients going to the ICU, a designated ICU team in full PPE receives the patient from the theatre anaesthesiology team at the exit between the yellow zone and the fast-track elevator. The patient is transferred on the theatre bed and moved to the ICU bed. The ward patients are initially transferred to an isolation room in the recovery area, where they are monitored until deemed safe to return to the ward. The recovery staff consisting of one nurse and one anaesthesiologist in full PPE receive the patient from the theatre anaesthesiology team in the sluice between the yellow zone and the corridor leading to the recovery room. In the case of short procedures when a quick recovery is expected, patients are fully recovered in the OR itself in order to minimise in-hospital transfer. After handing over the patient, the anaesthetic team doff following the same rules as the surgical team. All transfer routes are excluded from normal use until decontaminated.

If during a surgical procedure the protective barrier of any staff member allocated in the red or yellow zone is breached, they have to leave the area according to the aforementioned protocol. They need to contact the infectious control team for further guidance and are replaced by another staff member from the green zone if required.

Finally, the cleaning team in full PPE conducts thorough decontamination of all surface areas in the COA, starting from the red zone and finishing in the yellow zone. All unused disposable equipment is assumed to be contaminated and discarded into clinical waste bags. Surgical instruments are appropriately packed and sent for sterilisation. It is crucial to dispose of all waste, including the cling film which covers the equipment, to the red waste bags. The waste bags should be covered in additional waste bags and transported via the waste lifts all at the same time.

In summary, because theatre staff are at an increased risk of COVID-19 infection, strict and meticulous measures have been put in place to minimise the risk of cross infection. Although current guidelines provide clear and straightforward instructions [13], it is pivotal to adjust the operating facilities to their standards so that they can be fully implemented 
providing safety to the operating theatre personnel.

\section{Acknowledgments}

The authors would like to acknowledge the great contribution of the Operating Department Personnel for their dedication in developing and testing the protocol, and providing valuable opinions, hints, and advice. A special thank you to the following contributors: Elżbieta Białka-Mnich, Katarzyna Braszczyk, Magdalena Dryja, Małgorzata Gądek, Wioletta Kłosowska, Joanna Praszałowicz, Kinga Salawa, Wojciech Siuta, Wojciech Stycuła, Rafał Świstek, Ewa Lasota, and Edyta Twardowska.

\section{Conflict of interest}

The authors declare no conflict of interest.

\section{References}

1. World Health Organization. Naming the coronavirus disease (COVID-19) and the virus that causes it. Available from: https:// bit.ly/34CxdZv. Accessed 10th of April 2020.

2. Huang C, Wang Y, Li X, et al. Clinical features of patients infected with 2019 novel coronavirus in Wuhan, China. Lancet 2020; 395: 497-506.

3. Wang C, Horby PW, Hayden FG, et al. A novel coronavirus outbreak of global health concern. Lancet 2020; 395: 47-3.

4. https://coronavirus.jhu.edu/map.html. Accessed $10^{\text {th }}$ of April 2020.

5. Tien HC, Chughtai T, Jogeklar A, et al. Elective and emergency surgery in patients with severe acute respiratory syndrome (SARS). Can J Surg 2005; 48: 71-4.

6. Brindley P, Cardinal P. Optimizing Crisis Resource Management to Improve Patient Safety and Team Performance. Royal College of Physicians and Surgeons of Canada. $1^{\text {st }}$ Edition. May $15^{\text {th }}$ 2017. ISBN: 978-1-926588-40-7.

7. SAGES and EAES Recommendations Ragarding Surgical Res ponse to COVID-19 Crisis. 30/03/2020. Available from: https:// WwW.sages.org/recommendations-surgical-responsecovid-19/. Accessed 10 th of April 2020.

8. Balibrea JM, Badia JM, Rubio Pérez I, et al. Surgical management of patients with COVID-19 Infection. Recommendations of the Spanish Association of Surgeons. Cir Esp 2020 Apr 3. doi: 10.1016/j.ciresp.2020.03.001.

9. Brat G, Hersey S, Chhabra K, et al. Protecting surgical teams during the COVID-19 outbreak: a narrative review and clinical considerations. Ann Surg 2020 [Ahead of Print].

10. Coccolini F, Perrone G, Chiarugi M, et al. Surgery in COVID-19 patients: operational directives. World J Emerg Surg 2020; 15: 25.

11. Lasek A, Pędziwiatr M, Wysocki $M$, et al. Risk factors for intraabdominal abscess formation after laparoscopic appendectomy - results from the Pol-LA (Polish Laparoscopic Appendectomy) multicenter large cohort study. Videosurgery Miniinv 2019; 14: 70-8.
12. Piatkowski J, Jackowski M, Szeliga J. Laparoscopic surgery of esophageal hiatus hernia - single center experience. Videosurgery Miniinv 2014; 9: 13-7.

13. Mitura K, Myśliwiec P, Rogula W, et al. Wytyczne postępowania w oddziałach zabiegowych szpitali niejednoimiennych podczas pandemii COVID-19. Available from: https://tchp.pl. Accessed $17^{\text {th }}$ of April 2020.

Received: 12.04 .2020 , accepted: 28.04.2020. 